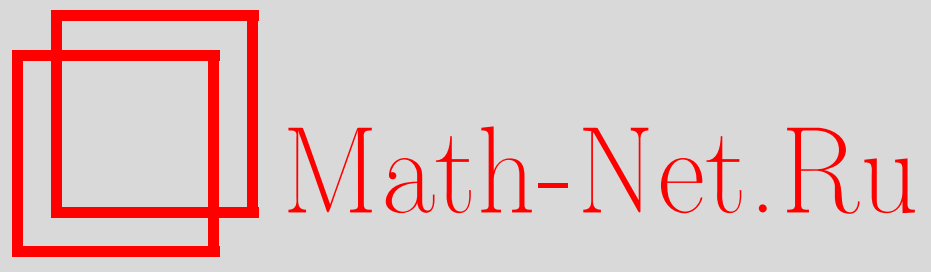

П. С. Салтыков, О связи топологической энтропии и энтропийной размерности, Матем. заметки, 2009, том 86, выпуск 2, 280-289

DOI: https://doi.org/10.4213/mzm4007

Использование Общероссийского математического портала Math-Net.Ru подразумевает, что вы прочитали и согласны с пользовательским соглашением http://www . mathnet.ru/rus/agreement

Параметры загрузки:

IP: 54.196 .121 .252

26 апреля 2023 г., 18:00:21

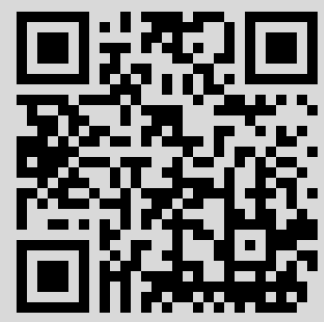


Том 86 выпуск 2 август 2009

\section{О связи топологической энтропии и энтропийной размерности}

\section{П. С. Салтыков}

Для липшицева отображения метрического компакта в себя имеется классическая верхняя оценка на топологическую энтропию - произведение энтропийной размерности компакта на логарифм константы Липшица. Гипотеза Жиса состоит в том, что, варьируя метрику, можно сколь угодно точно приблизить верхнюю оценку к точному значению топологической энтропии. В настоящей работе получен критерий выполнения гипотезы Жиса для индивидуального отображения. Применив этот критерий, мы доказываем гипотезу Жиса для гиперболических отображений.

Библиография: 5 названий.

\section{1. Введение}

Топологическая энтропия была введена в 1965 г. в работе [1] как один из способов измерять "сложность" отображения. Для гладких отображений компактных многообразий ее конечность была установлена теоремой Кушнеренко (см. [2; с. 37-38]), дающей эффективную оценку для $f: M \rightarrow M$ как

$$
h_{\text {top }}(f) \leqslant \operatorname{dim} M \cdot \ln \left\|f^{\prime}\right\| .
$$

Эта оценка обобщается до следующей:

$$
h_{\text {top }}(f) \leqslant \operatorname{dim}_{E} X \cdot \ln L(f),
$$

где $f: X \rightarrow X-$ липшицево отображение метрического компакта в себя, $\operatorname{dim}_{E} X-$ энтропийная размерность, а через $L(f)$ обозначена константа Липшица отображения $f$. (Определения топологической энтропии и энтропийной размерности напоминаются в разделе 2.)

Рассмотрим на компакте $X$ класс метрик, порождающих одну и ту же топологию. Обозначим этот класс через D. Топологическая энтропия, как следует из ее названия, определяется исключительно топологией на $X$ (впрочем, зачастую оказывается удобнее работать с использующим метрику определением, которое мы напоминаем ниже; независимость результата от выбора метрики из $\mathbf{D}$ доказывается, например, в [3; с. 121]). В результате оценка (1) может быть улучшена следующим образом:

$$
h_{\mathrm{top}}(f) \leqslant \inf _{d \in \mathbf{D}} \operatorname{dim}_{E}(X, d) \cdot \ln L_{d}(f) .
$$

В связи с этим Э. Жисом была сформулирована следующая гипотеза.

Работа выполнена при поддержке совместного гранта Российского фонда фундаментальных исследований и фонда CNRS (грант № 05-01-02801).

(C) П. С. САлтыков, 2009 
ГиПотезА. Пусть $f: X \rightarrow X$ - липшищево отображение метрического компакта $X$ с конечной энтропийной размерностью в себя. В этом случае неравенство в (2) можно заменить на равенство.

Настоящая работа посвящена исследованию необходимых и достаточных условий того, что для отображения $f$ выполняется гипотеза Жиса. Показано, что для гиперболических отображений гипотеза Жиса верна. При доказательстве мы используем следующий критерий.

Теорема 1 (см. определение 5 в разделе 3). Гипотеза Жиса выполняется тогда u только тогда, когда сходимость в определении $h_{\mathrm{top}}$ экспоненциальная.

Затем мы проверяем, что гиперболические отображения удовлетворяют этому критерию.

\section{2. Обозначения и определения}

Пусть

- $f: X \rightarrow X$ - липшицево отображение метрического компакта $X$ в себя;

- $d$ - метрика на компакте $X$;

- $L_{d}(f)$ - константа Липшица для $f$ в метрике $d$.

ОПРЕДЕЛЕНИЕ 1. Рассмотрим метрику

$$
d_{N}(x, y)=\max _{0 \leqslant k<N-1} d\left(f^{k}(x), f^{k}(y)\right)
$$

на $X$. Тогда $\varepsilon$-сеть для метрики $d_{N}$ называется $(N, \varepsilon)$-сетъю для метрики $d$.

Обозначим через $\Sigma(N, \varepsilon)$ мощность минимальной $(N, \varepsilon)$-сети.

Введем функцию

$$
h(\varepsilon)=\varlimsup_{n \rightarrow \infty} \frac{\ln \Sigma(n, \varepsilon)}{n} .
$$

ОПРЕДЕЛЕНИЕ 2. Топологической энтропией называется число

$$
h_{\mathrm{top}}(f)=\lim _{\varepsilon \rightarrow+0} h(\varepsilon) .
$$

Пусть $S(r)$ - мощность минимальной $r$-сети в метрике $d$.

ОПРЕДЕЛЕНИЕ 3. Энтропийная размерность метрического пространства $X$ определяется как

$$
\operatorname{dim}_{E} X=\lim _{r \rightarrow+0} \frac{\ln S(r)}{|\ln r|} .
$$

Будем ее также обозначать через $\operatorname{dim}_{E}(X, d)$, явно указывая зависимость от метрики.

\section{3. Основной результат}

\section{1. Формулировка результатов.}

ОПРЕДЕлЕНИЕ 4. Сходимость в определении $h_{\mathrm{top}}(f)$ экспоненциалъная, если для любого $\delta>0$ существуют метрика $d_{\delta}$, топологически эквивалентная исходной мет- 
рике $d$, и константы $C$ и $\varepsilon_{\delta}>0$ такие, что для любого $0<\varepsilon<\varepsilon_{\delta}$ для $N>C \cdot|\ln \varepsilon|$ верно неравенство

$$
\frac{\ln \left(\Sigma_{d_{\delta}}(N, \varepsilon)\right)}{N} \leqslant h_{\mathrm{top}}(f)+\delta .
$$

Поскольку на протяжении данной работы отображение $f$ варьироваться не будет, для краткости мы зачастую будем писать просто $L_{d}$ вместо $L_{d}(f)$ и $h_{\text {top }}$ вместо $h_{\text {top }}(f)$.

Теорема 2 (критерий сходимости для гипотезы Жиса). Для выполнения равенcmвa

$$
h_{\mathrm{top}}(f)=\inf _{d \in \mathbf{D}} \operatorname{dim}_{E}(X, d) \cdot \ln L_{d}(f)
$$

необходимо и достаточно, чтобы сходимость в определении $h_{\text {top }}$ была экспоненциальной.

СлЕДСТВИЕ 1. Для гиперболических отображений гипотеза Жиса верна.

3.2. План доказательства теоремы и следствия. Для доказательства достаточности (п. 4.1) мы в явном виде предъявим семейство метрик $m_{\lambda}$ такое, что

$$
\ln \left(L_{m_{\lambda}}(f)\right) \cdot \operatorname{dim}_{E}\left(X, m_{\lambda}\right) \underset{\lambda \rightarrow 1}{\longrightarrow} h_{\mathrm{top}}(f) .
$$

При построении этого семейства мы будем использовать расстояния $d\left(f^{k}(x), f^{k}(y)\right)$. Липшицевость будет получаться автоматически, а экспоненциальная сходимость в определении топологической энтропии обеспечит нужную оценку на энтропийную размерность.

Необходимость (п. 4.2) доказывается рассуждениями, аналогичными доказательству теоремы Кушнеренко, и явным построением константы $C$, требуемой в определении экспоненциальной сходимости.

В разделе 5 доказывается, что для гиперболических (а на самом деле и для внутренне-гиперболических, см. определение там же) отображений условие экспоненциальной сходимости в определении топологической энтропии выполнено. Значит, такие отображения удовлетворяют гипотезе Жиса. Экспоненциальная сходимость следует из явной оценки на мощность $\Sigma(N, \varepsilon)$-сети.

\section{4. Доказательство основного результата}

Случай $L_{d}=1$ или $\operatorname{dim}_{E}(X, d)=0$ очевиден, поэтому далее будем предполагать, что $L_{d}>1$ и $\operatorname{dim}_{E}(X, d)>0$. Без ограничения общности можно считать, что $\operatorname{diam} X=1$.

4.1. Достаточность. Фиксируем $d \in \mathbf{D}$. Напомним, что $\Sigma(N, \varepsilon)-$ мощность минимальной $(N, \varepsilon)$-сети. Для любого $\lambda>0$ рассмотрим метрику

$$
m_{\lambda}(x, y)=\sum_{k \geqslant 0} \lambda^{k} \cdot d\left(f^{k}(x), f^{k}(y)\right) .
$$

Легко видеть, что при любом $\lambda \in(0,1)$ ряд сходится.

ЛЕмма 1. Для любого $\lambda \in(0,1)$ метрика $m_{\lambda}$ топологически эквивалентна исходной метрике $d$. 
Доказательство. Утверждение вытекает из неравенств $m_{\lambda} \geqslant d, \operatorname{diam} X<\infty$ и непрерывности $f$.

Лемма 2. Константа Липшица для $f$ в метрике $m_{\lambda}$ допускает следующую оценку:

$$
L_{m_{\lambda}} \leqslant \frac{1}{\lambda}
$$

ДокАЗАТЕЛЬСтво. Проведем прямые вычисления. Имеем

$$
\begin{aligned}
m_{\lambda}(f(x), f(y)) & =\sum_{k \geqslant 0}\left(\lambda^{k} \cdot d\left(f^{k+1}(x), f^{k+1}(y)\right)\right) \\
& =\frac{1}{\lambda} \cdot\left(m_{\lambda}(x, y)-d(x, y)\right) \leqslant \frac{1}{\lambda} \cdot m_{\lambda}(x, y) .
\end{aligned}
$$

Значит,

$$
L_{m_{\lambda}}=\sup _{x, y \in X} \frac{m_{\lambda}(f(x), f(y))}{m_{\lambda}(x, y)} \leqslant \frac{1}{\lambda} \cdot \frac{m_{\lambda}(x, y)}{m_{\lambda}(x, y)}=\frac{1}{\lambda} .
$$

Пусть $S_{\lambda}(r)$ - мощность минимальной $r$-сети в метрике $m_{\lambda}$.

Лемма 3. Рассмотрим $(N, \varepsilon)$-сеть для $f$ в метрике $d$ (состоящую из $\Sigma(N, \varepsilon)$ точек). Тогда она одновременно является $r_{\delta}$-сетью в метрике $m_{\lambda}$, где

$$
r_{\delta}:=r_{\lambda}(N, \varepsilon)=\frac{\varepsilon+\lambda^{N}}{1-\lambda} .
$$

ДокАЗАтЕЛЬство. Рассмотрим произвольную точку $x$, пусть $y$ - ближайшая к ней точка $(N, \varepsilon)$-сети согласно метрике $d_{N}$. Оценим расстояние между ними в метрике $m_{\lambda}$ :

$$
\begin{aligned}
m_{\lambda}(x, y) & \leqslant \sum_{k \geqslant 0}^{N-1} \lambda^{k} \cdot d\left(f^{k}(x), f^{k}(y)\right)+\sum_{k=N}^{\infty} \lambda^{k} \cdot d\left(f^{k}(x), f^{k}(y)\right) \\
& \leqslant \sum_{k \geqslant 0}^{N-1} \lambda^{k} \cdot \varepsilon+\sum_{k=N}^{\infty} \lambda^{k} \cdot 1 \leqslant \frac{1-\lambda^{N}}{1-\lambda} \cdot \varepsilon+\frac{\lambda^{N}}{1-\lambda} \leqslant \frac{\varepsilon+\lambda^{N}}{1-\lambda} .
\end{aligned}
$$

СлЕДСТВИЕ 2. Для любого $r>0 u \lambda \in(0,1)$ положим

$$
\varepsilon_{\lambda}(r)=\frac{1}{2} \cdot r \cdot(1-\lambda), \quad N_{\lambda}(r)=\left[\frac{\left|\ln \left(\varepsilon_{\lambda}(r)\right)\right|}{|\ln \lambda|}\right]+1,
$$

где $[x]$ - иелая часть числа $x$. Тогда

$$
S_{\lambda}(r) \leqslant \Sigma\left(N_{\lambda}(r), \varepsilon_{\lambda}(r)\right) .
$$

ДокАзАтЕЛьство. Как следует из леммы 3 ,

$$
S\left(r_{\lambda}(N, \varepsilon)\right) \leqslant \Sigma(N, \varepsilon), \quad \text { где } \quad r_{\lambda}(N, \varepsilon)=\frac{\varepsilon+\lambda^{-N}}{1-\lambda} .
$$

Но функции $\varepsilon_{\lambda}(r)$ и $N_{\lambda}(r)$ были выбраны так, что $r_{\lambda}\left(N_{\lambda}(r), \varepsilon_{\lambda}(r)\right) \leqslant r$ (что легко проверяется прямой выкладкой). Подставляя $\varepsilon=\varepsilon_{\lambda}(r)$ и $N=N_{\lambda}(r)$ в $(7)$, получаем требуемое заключение. 
Утверждения, сформулированные и доказанные выше, верны для любой метрики $d$ из D. Дальше мы будем пользоваться условием экспоненциальной сходимости.

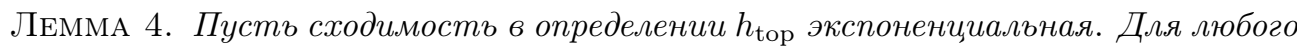
$\delta>0$ рассмотрим соответствуюшую ему метрику $d_{\delta}$ в смысле определения 4. Тогда существуют $\lambda, r_{0}$ такие, что для всех $r<r_{0}$ выполнено

$$
\begin{aligned}
& \text { 1) } \frac{\ln \Sigma\left(N_{\lambda}(r), \varepsilon_{\lambda}(r)\right)}{N_{\lambda}(r)} \leqslant h_{\text {top }}+\delta, \\
& \text { 2) } \frac{N_{\lambda}(r)}{|\ln r|} \leqslant \frac{1+\delta}{|\ln \lambda|},
\end{aligned}
$$

где $N_{\lambda}$ и $\varepsilon_{\lambda}$ выбираются согласно (5).

Доказательство. Докажем (8). Неравенство (8) - это неравенство (3) при $N=$ $N_{\lambda}(r)$ и $\varepsilon=\varepsilon_{\lambda}(r)$. По определению 1 для его выполнения достаточно неравенства

$$
N_{\lambda}(r) \geqslant C \cdot\left|\ln \varepsilon_{\lambda}(r)\right| .
$$

Введем величину

$$
N_{\lambda}^{1}(r)=\frac{\left|\ln \left(\varepsilon_{\lambda}(r)\right)\right|}{|\ln \lambda|} .
$$

Учитывая, что $N_{\lambda}^{1} \leqslant N_{\lambda}$, для (10) достаточно выполнения неравенства $1 /|\ln \lambda| \geqslant C$. По данному $C$ всегда можно выбрать $\lambda$, столь близкое к 1 , что последнее неравенство выполнено. Таким образом, (8) доказано.

Фиксируем какое-либо $\lambda$, удовлетворяющее (8). Тогда

$$
\frac{N_{\lambda}(r)}{|\ln r|}=\frac{N_{\lambda}^{1}(r)}{|\ln r|} \cdot \frac{N_{\lambda}(r)}{N_{\lambda}^{1}(r)}=\left|\frac{\ln (r \cdot(1-\lambda) / 2)}{\ln r \cdot \ln \lambda}\right| \cdot \frac{N_{\lambda}(r)}{N_{\lambda}^{1}(r)}
$$

(второе равенство следует из (5)).

Подставим это выражение в (9), получим

$$
\left|\frac{\ln (r \cdot(1-\lambda) / 2)}{\ln r}\right| \cdot \frac{N_{\lambda}(r)}{N_{\lambda}^{1}(r)} \leqslant 1+\delta .
$$

Ясно, что при $r \rightarrow+0$ левая часть в (11) стремится к 1 . Значит, существует $r_{0}>0$ такое, что выполнено (9).

Лемма 4 доказана.

Окончание доказательства достаточности. По заданному $\delta$ находим метрику $d_{\delta}$ и константу $C$ по определению 4. По этому $C$, используя следствие 1 и лемму 4 , находим $\lambda$ такое, что выполнены (8) и (9) для $r<r_{0}$, где $N_{\lambda}(r)$ и $\varepsilon_{\lambda}(r)$ выбираются согласно (5). Применяя последовательно следствие 2 и лемму 4 , получим

$$
\begin{aligned}
\frac{\ln S_{\lambda}(r)}{|\ln r|} & \leqslant \frac{\ln \Sigma\left(N_{\lambda}(r), \varepsilon_{\lambda}(r)\right)}{N_{\lambda}(r)} \cdot \frac{N_{\lambda}(r)}{|\ln r|} \\
& \leqslant\left(h_{\mathrm{top}}+\delta\right) \cdot \frac{1+\delta}{|\ln \lambda|} \leqslant \frac{1}{|\ln \lambda|} \cdot\left(h_{\mathrm{top}}+\delta \cdot\left(1+h_{\mathrm{top}}+\delta\right)\right) .
\end{aligned}
$$

Обозначим $\delta \cdot\left(1+h_{\mathrm{top}}+\delta\right)$ через $\delta^{1}$. Тогда $\delta^{1} \rightarrow 0$ при $\delta \rightarrow 0$. 
Домножим левую часть последнего неравенства на $\ln L_{m_{\lambda}}$, а правую на $\ln (1 / \lambda)$. При этом по лемме 2 неравенство сохранится. Получим

$$
\frac{\ln S_{\lambda}(r)}{|\ln r|} \cdot \ln L_{m_{\lambda}} \leqslant h_{\text {top }}+\delta^{1} .
$$

Это неравенство верно для всех $r<r_{0}$. Перейдем в нем к пределу при $r \rightarrow+0$. Получим необходимое неравенство

$$
\operatorname{dim}_{E}\left(X, m_{\lambda}\right) \cdot \ln L_{m_{\lambda}} \leqslant h_{\text {top }}+\delta^{1} .
$$

Для каждого $\delta^{1}>0$ мы предъявили метрику $m_{\lambda}$ такую, что выполнено неравенство (12). Перейдем к точной нижней грани по $\delta^{1}$ при $\delta^{1} \rightarrow+0$. Получим

$$
\inf _{\lambda}\left(\operatorname{dim}_{E}\left(X, m_{\lambda}\right) \cdot \ln L_{m_{\lambda}}\right) \leqslant h_{\mathrm{top}},
$$

что вместе с (1) завершает доказательство достаточности.

4.2. Необходимость. Пусть выполнено равенство (4). Тогда для любого $\delta>0$ существует метрика $d(\delta)$ из $\mathbf{D}$ такая, что

$$
\ln L_{d(\delta)} \cdot \operatorname{dim}_{E}(X, d(\delta))<h_{\text {top }}+\delta .
$$

Будем считать без ограничения общности, что $\delta<1$. Фиксируем произвольное $\delta \in(0,1)$ и метрику $d(\delta)$ из $\mathbf{D}$, удовлетворяющую $(14)$. Обозначим $L_{d(\delta)}$ через $L$.

Приведенные ниже рассуждения аналогичны доказательству теоремы Кушниренко о конечности энтропии. Заметим, что

$$
\Sigma(N, \varepsilon) \leqslant S\left(L^{-N} \varepsilon\right)
$$

Поскольку $\ln S(r) /|\ln r| \rightarrow \operatorname{dim}_{E}(X, d(\delta))$ и верно (14), то, начиная с некоторого $r_{0}$, имеем для всех $r \leqslant r_{0}$

$$
\frac{\ln S(r)}{|\ln r|} \cdot \ln L<h_{\text {top }}+\delta .
$$

Подставим в последнее неравенство оценку (15). Тогда для любого $N$ такого, что $L^{-N} \varepsilon \leqslant r_{0}$, выполнено

$$
\frac{\ln \Sigma(N, \varepsilon)}{N \ln L+\ln \varepsilon^{-1}} \cdot \ln L=\frac{\ln \Sigma(N, \varepsilon)}{N} \cdot \frac{1}{1+(1 / N) \ln (L / \varepsilon)}<h_{\text {top }}+\delta .
$$

Следовательно, при выполнении условий

$$
L^{-N} \varepsilon \leqslant r_{0}, \quad 1+\frac{1}{N} \ln \frac{L}{\varepsilon}<\frac{h_{\text {top }}+2 \delta}{h_{\text {top }}+\delta}
$$

имеем

$$
\frac{\ln \Sigma(N, \varepsilon)}{N}<h_{\text {top }}+2 \delta .
$$

Первое из условий равносильно

$$
N \geqslant \frac{\ln r_{0}+\ln \varepsilon^{-1}}{\ln L}=C_{1}+C_{2} \ln \varepsilon^{-1},
$$


а второе -

$$
N>\frac{h_{\text {top }}+\delta}{\delta} \cdot \ln \frac{L}{\varepsilon}=C_{3}+C_{4} \ln \varepsilon^{-1} .
$$

Оба условия выполняются, начиная с $N_{0}=C_{5} \ln \varepsilon$. Получаем, что для любого $\varepsilon>0$, $\varepsilon<1 / 2$, для всех $N>N_{0}=C_{5} \ln \varepsilon^{-1}$ верно (16). В силу произвольности $\delta$ по определению имеем экспоненциальную сходимость в определении топологической энтропии.

Теорема доказана полностью.

4.3. Пример. В этом пункте мы приведем пример отображения с нулевой топологической энтропией, для которого в определении энтропии относительно стандартной метрики сходимость неэкспоненциальная. Однако, так как заменой метрики она может быть сделана экспоненциальной, контрпримером к гипотезе Жиса этот пример не является.

Рассмотрим пространство Бернулли двоичных последовательностей

$$
\Sigma_{2}^{+}=\left\{x_{1}, x_{2}, x_{3}, \ldots \mid x_{I} \in\{0,1\}\right\}, \quad d(x, y)=2^{-\min _{i}\left(x_{i} \neq y_{i}\right)} .
$$

Выберем (очень быстрорастущую последовательность) $b_{j}, j \in \mathbb{N}$, и пусть $s_{j}=1+$ $\sum_{l=1}^{j-1} b_{j}$. Заметим, что $\Sigma_{2}^{+}-$компакт с конечной энтропийной размерностью.

На этом множестве введем отображение $f$ следующим образом. На каждом $j$-м участке длиной $b_{j}$ действует левый сдвиг, а выпадающие справа символы заменятся нулями. Более точно:

$$
\underbrace{x_{1} \ldots x_{b_{1}}}_{b_{1}} \underbrace{x_{s_{2}} \ldots x_{s_{2}+b_{2}-1}}_{b_{2}} x_{s_{3}} \ldots \mapsto \underbrace{x_{2} \ldots x_{b_{1}} 0}_{b_{1}} \underbrace{x_{s_{2}+1} \ldots x_{s_{2}+b_{2}-1} 0}_{b_{2}} x_{s_{3}+1} \ldots
$$

Легко видеть, что для этого отображения $h_{\mathrm{top}}=0$, так как все точки стремятся к нулевой последовательности. Выберем какое-то натуральное $j$ и рассмотрим $\varepsilon=$ $2^{-s_{j}}$. Тогда $\Sigma(N, \varepsilon)=2^{s_{j}} \cdot 2^{N}$ при $0 \leqslant N<b_{j}$. Значит, при $N \leqslant b_{j}$

$$
\frac{\ln \Sigma(N, \varepsilon)}{N}=\frac{s_{j}+N}{N} \cdot \ln 2 .
$$

Теперь зафиксируем выбор последовательности $b_{j}$ : потребуем, чтобы $b_{j} / s_{j} \rightarrow \infty$ при $j \rightarrow \infty$. Например, рассмотрим $b_{j}=e^{j^{2}}$.

Легко видеть, что для любой заданной наперед константы $C$ при $\varepsilon=2^{-s_{j}}$ и $N \sim C|\ln \varepsilon| \sim C^{\prime} s_{j}$ имеем $N \leqslant b_{j}$ и, значит,

$$
\frac{\ln \Sigma(N, \varepsilon)}{N}=\frac{s_{j}+2 C \cdot s_{j}-1}{2 C \cdot s_{j}} \cdot \ln 2 \geqslant \ln 2 .
$$

Это и означает, что сходимость в пределе для энтропии (равной 0) неэкспоненциальная в смысле исходной метрики. Однако добиться выполнения условия экспоненциальной сходимости в этом примере можно. По заданной последовательности $b_{j}$ выберем достаточно быстрорастущую последовательность $k_{j}$, например, положив $k_{j}=s_{j+1}^{2}$. По этой последовательности построим отображение "вписывания нулей" $x \mapsto \widetilde{x}$, определяемое так:

$$
\begin{aligned}
& x_{1} \ldots x_{b_{1}} x_{s_{2}} \ldots x_{s_{2}+b_{2}-1} x_{s_{3}} \ldots \\
& \quad \mapsto \quad x_{1} \ldots x_{b_{1}} \underbrace{0 \ldots 0}_{k_{1}} x_{s_{2}+k_{1}} \ldots x_{s_{2}+b_{2}+k_{1}}-1 \underbrace{0 \ldots 0}_{k_{2}} x_{s_{3}+k_{1}+k_{2}}^{0 \ldots}
\end{aligned}
$$


Положим $\widetilde{d}(x, y)=d(\widetilde{x}, \widetilde{y})$. Нетрудно доказать, что метрики $d$ и $\widetilde{d}$ топологически эквивалентны. Прямыми вычислениями устанавливается, что при подходящем выборе констант $k_{1}, k_{2} \ldots$, например, таком, как указано выше, условие экспоненциальной сходимости выполняется.

\section{5. Доказательство гипотезы Жиса для гиперболических гомеоморфизмов}

Во всем разделе предполагаем, что компакт $X$ с метрикой $d$ и отображение $f$ фиксированы. Поэтому для упрощения обозначений мы не будем явно указывать зависимости переменных от них. Будем предполагать, что $\operatorname{dim}_{E} X>0$, так как случай $\operatorname{dim}_{E} X=0$ тривиален.

5.1. Подготовительные сведения из гиперболической теории. Мы напомним несколько определений и известных фактов из теории гиперболических отображений, которые могут быть найдены, например, в [3] и [4].

ОПРЕДЕЛЕНИЕ 5. Гомеоморфизм метрического компакта $X$ с метрикой $d$ называется разделяющим, если существует константа $\delta_{0}>0$ такая, что для любых точек $x$, $y$ из условия

$$
d\left(f^{n}(x), f^{n}(y)\right)<\delta_{0} \quad \text { для всех } n \in \mathbb{Z}
$$

следует, что $x=y$. Верхняя грань таких чисел $\delta_{0}$ называется постоянной разделения.

Пусть $(\Lambda, d)$ - компактное метрическое пространство, $f: \Lambda \rightarrow \Lambda$ - липшицеморфизм (т.е. $f$ - гомеоморфизм, $f$ и $f^{-1}$ липшицевы). Определим два отношения эквивалентности $\sim_{u}$ и $\sim_{s}$ на $\Lambda$ следующим образом:

$$
\begin{aligned}
& x \sim_{u} y \quad \Longleftrightarrow d\left(f^{-n}(x), f^{-n}(y)\right) \underset{n \rightarrow \infty}{\longrightarrow} 0, \\
& x \sim_{s} y \quad \Longleftrightarrow d\left(f^{n}(x), f^{n}(y)\right) \underset{n \rightarrow \infty}{\longrightarrow} 0 .
\end{aligned}
$$

Определим также локально устойчивое и неустойчивое множества:

$$
\begin{aligned}
& W_{\epsilon}^{\mathrm{s}}(x)=\left\{z \mid z \sim_{s} x \text { и } d\left(f^{n}(x), f^{n}(z)\right) \leqslant \epsilon \text { для всех } n \in \mathbb{N}^{+}\right\}, \\
& W_{\epsilon}^{\mathrm{u}}(x)=\left\{z \mid z \sim_{u} x \text { и } d\left(f^{-n}(x), f^{-n}(z)\right) \leqslant \epsilon \text { для всех } n \in \mathbb{N}^{+}\right\} .
\end{aligned}
$$

ОпредЕЛЕНиЕ 6. Липшицеморфизм $f$ компактного метрического пространства $(\Lambda, d)$ называется внутренне-гиперболическим, если для некоторых $\vartheta>0, \epsilon>0$, выполнено следующее:

1) если $d(x, y)<\vartheta$, то существует единственная точка

$$
w(x, y)=W_{\epsilon}^{\mathrm{u}}(x) \cap W_{\epsilon}^{\mathrm{s}}(y) \in \Lambda
$$

отображение $(x, y) \mapsto w(x, y)$ непрерывно;

2) существуют константы $C>0,0<\nu<1,0<\xi<1$, не зависящие от точки, такие, что для всех точек компакта $X$ выполнено

$$
\begin{aligned}
y \in W_{\epsilon}^{\mathrm{s}}(x) & \Longrightarrow \quad d\left(f^{n}(x), f^{n}(y)\right) \leqslant C \nu^{n} d(x, y), \quad n \in \mathbb{N}, \\
y \in W_{\epsilon}^{\mathrm{u}}(x) & \Longrightarrow \quad d\left(f^{-n}(x), f^{-n}(y)\right) \leqslant C \xi^{n} d(x, y), \quad n \in \mathbb{N} .
\end{aligned}
$$


(Определение внутренне-гиперболического отображения впервые появилось в работе Алексеева и Якобсона [5], а данная формулировка взята из [4; с. 404].)

Следующая лемма легко выводится из компактности множества.

Лемма 5. Пусть $f: X \rightarrow X$-разделяющий гомеоморфизм с константой разделения $\delta_{0}$. Въберем и зафиксируем $\gamma<\delta_{0}$. Тогда для любого $\varepsilon>0$ существует натуральное $K=K(\varepsilon)$ такое, что из условия

$$
d\left(f^{k}(x), f^{k}(y)\right)<\gamma \quad \text { npu } \quad k=-K(\varepsilon), \ldots, K(\varepsilon)
$$

cледует, что $d(x, y)<\varepsilon$.

Из этой леммы мгновенно выводится утверждение, позволяющее оценить мощности $(N, \varepsilon)$-сетей при всех $\varepsilon$ через мощности сетей только вида $\left(N^{\prime}, \gamma\right)$.

СлЕДСТВиЕ 3. Имеет место оценка

$$
\Sigma(N, \varepsilon) \leqslant \Sigma(N+2 K(\varepsilon), \gamma)
$$

\section{2. Применение критерия сходимости для гиперболического случая.}

Теорема 3. Пусть $(\Lambda, d)$ - компактное метрическое пространство $и f: \Lambda \rightarrow$ $\Lambda$ - внутренне-гиперболическое отображение. Тогда для $f$ выполняется гипотеза Жиса, а именно,

$$
h_{\mathrm{top}}=\inf _{d} \operatorname{dim}_{E}(\Lambda, d) \cdot \ln L_{d}(f) .
$$

ДокАЗАТЕЛЬСТво. Предположим, что мы зафиксировали константу $\gamma_{0}<\vartheta$. В силу непрерывной зависимости $w(x, y)$ от $x$ и $y$ найдется такое $\varepsilon_{0}<\gamma_{0} / 2 C$, что при $d(x, y)<\varepsilon_{0}$ расстояния $d(x, w(x, y))$ и $d(y, w(x, y))$ не превосходят $\gamma_{0} / 2 C$. Нам потребуется следующая

ЛЕмма 6. Если выполнены условия теоремы 3, то $f$ является разделяющим отображением с некоторой постоянной разделения $\delta_{0}>\gamma_{0}$; более того, существует константа $D$ такая, что в качестве $K(\varepsilon)$ можно взять $[D|\ln \varepsilon|]$ для всех $\varepsilon<\varepsilon_{0}$.

ДокАЗАТЕЛЬСтво. Предположим, что $d(x, y)>\varepsilon$ (и при этом $\left.d(x, y)<\gamma_{0}\right)$. Тогда определена точка $w=w(x, y)$. Одно из расстояний $d(x, w)$ и $d(w, y)$ не меньше $\varepsilon / 2$. Если это $d(x, w)$, то не более чем за $\left[\log _{\xi} C^{-1} d(x, y) / 2 \gamma_{0}\right]+1$ итераций точки $x$ и $w$ разойдутся на расстояние, большее $\gamma_{0}$. При этом расстояние между образами $w$ и $y$ увеличится не более, чем в $C$ раз. Значит, в этот момент

$$
d\left(f^{k}(x), f^{k}(y)\right) \geqslant d\left(f^{k}(x), f^{k}(w)\right)-d\left(f^{k}(w), f^{k}(y)\right) \geqslant \gamma_{0}-\frac{C \gamma_{0}}{2 C} \geqslant \frac{\gamma_{0}}{2}
$$

вследствие выбора $\varepsilon_{0}$ и условия $\varepsilon<\varepsilon_{0}$. Аналогично разбирается случай, когда большим оказывается $d(w, y)$ (при этом итерируется $\left.f^{-1}\right)$. В каждом из случаев нам требуется не более, чем $D \ln \varepsilon^{-1}$ итераций.

Для доказательства теоремы 3 покажем, что выполняется условие теоремы 1, т.е. что сходимость экспоненциальная. В силу леммы 6 выполнено заключение следствия 3, следовательно,

$$
\Sigma(N, \varepsilon) \leqslant \Sigma(N+2 K(\varepsilon), \gamma)
$$


Заметим теперь, что

$$
\frac{\ln \Sigma(N, \varepsilon)}{N} \leqslant \frac{\ln \Sigma(N+2 K(\varepsilon), \gamma)}{N+2 K(\varepsilon)} \cdot \frac{N+2 K(\varepsilon)}{N} \leqslant \frac{\ln \Sigma(N+2 K(\varepsilon), \gamma)}{N+2 K(\varepsilon)} \cdot\left(1+\frac{C_{1}|\ln \varepsilon|}{N}\right) .
$$

Поскольку предел по $\varepsilon$ в определении топологической энтропии на самом деле берется от монотонно неубывающей функции, легко видеть, что для любого $\delta>0$ найдется $N_{0}$ такое, что при всех $N>N_{0}$ выполнено неравенство

$$
\frac{\ln \Sigma(N, \gamma)}{N} \leqslant h_{\text {top }}+\delta
$$

Тогда из $N>N_{0}$ и

$$
\left(1+\frac{C_{1}|\ln \varepsilon|}{N}\right)<\frac{h_{\mathrm{top}}+2 \delta}{h_{\mathrm{top}}+\delta}
$$

следует, что

$$
\frac{\ln \Sigma(N, \varepsilon)}{N} \leqslant\left(h_{\mathrm{top}}+\delta\right) \cdot\left(\frac{h_{\mathrm{top}}+2 \delta}{h_{\mathrm{top}}+\delta}\right)=h_{\mathrm{top}}+2 \delta .
$$

Условие (18) переписывается как

$$
N \geqslant \frac{D|\ln \varepsilon| \cdot\left(h_{\mathrm{top}}+\delta\right)}{\delta}=D_{1}|\ln \varepsilon|
$$

и в силу произвольности $\delta>0$ неравенство (19) доказывает экспоненциальную скорость сходимости в определении топологической энтропии.

Теорема 3 доказана.

Автор благодарен Э. Жису за постановку задачи и интерес к работе; также автор выражает свою признательность Ю. С. Ильяшенко, Т. И. Голенищевой-Кутузовой и В. А. Клепцыну за полезные обсуждения и помощь в подготовке настоящей статьи.

\section{СПИСОК ЦИТИРОВАННОЙ ЛИТЕРАТУРЫ}

[1] R. L. Adler, A. G. Konheim, M. H. McAndrew, "Topological entropy", Trans. Amer. Math. Soc., 114 (1965), 309-319.

[2] А. Г. Кушниренко, "Оценка сверху энтропии классической динамической системы", Докл. АН СССР, 161:3 (1965), 37-38.

[3] А.Б. Каток, Б. Хасселблат, Введение в современную теорию динамических систем, Факториал, М., 1999.

[4] Ю. С. Ильяшенко, В. Ли, Нелокальные бифуркации, Новые математические дисциплины, МЦНМО, М., 1999.

[5] V. M. Alekseev, M. V. Yakobson, "Symbolic dynamics and hyperbolic dynamic systems", Phys. Rep., 75:5 (1981), 287-325.

П. С. Салтыков

Московский государственный университет им. М. В. Ломоносова

E-mail: p_saltykov@pochta.ru
Поступило

24.10 .2006

Исправленный вариант

12.05 .2008 